\title{
Effect of Darling-Dennison and Fermi Resonance on Thermodynamic Functions
}

\author{
Harold W. Woolley
}

\begin{abstract}
The effect on thermodynamic functions due to resonance of the Darling-Dennison type for water and of the Fermi type for carbon dioxide is calculated working directly with the elements of the secular determinants that define the energy levels.
\end{abstract}

\section{Introduction}

In calculating thermodynamic functions for various molecular systems, the complication is sometimes encountered that the energy levels are expressed indirectly by means of a determinantal equation. In many cases, the determinant will have nonzero elements only along and quite near to the principal diagonal. Frequently, no explicit general solution for the positions of the levels as affected by the off-diagonal elements may be known, so that an explicit determination of the related thermodynamic properties as influenced by the effect in question would seem difficult at best. In some of these cases, useful results can be obtained in a general form from the secular equation without making use of explicit individual solutions.

\section{Secular Determinant Method}

If the secular determinant, $\left|E_{i j}-\delta_{i j} W\right|$, is expanded in polynomial form, beginning with the highest power of the unknown, $W$, then the successive coefficients for the different powers of $W$ give the sum of roots and sums of products of the roots, such as $\underset{i}{\Sigma} W_{i}, \sum_{i<j} \sum_{i} W_{j}, \underset{i<j<k}{\Sigma \Sigma} W_{i} W_{j} W_{k}$, etc., when allowance is made for alteration of signs in the successive terms.

In the calculation of thermodynamic functions, the intermediate step of obtaining partition functions involves the evaluation and addition of a large number of Boltzmann factors, using the energies of the various levels. This factor, $e^{-W}{ }_{i}{ }^{l k T}$, is expressible as a series so that the summation over a set of energy levels

$$
\sum_{i} e^{-W_{i} / k T}=\sum_{m=0} \frac{(-k T)^{-m}}{m !} \sum_{i} W_{i}^{m}
$$

may be found if the individual sums of powers of energy $\Sigma W_{i}, \Sigma W_{i}^{2}, \Sigma W_{i}^{3}$, etc., are known. It is possible to find these quantities in terms of sums of products of the roots and hence in terms of the coefficients of the polynomial expansion of the secular determinant. In this process several algebraic steps are involved including the application of the multinomial theorem. The result can thus be put in the form

$$
\begin{gathered}
\sum_{i} W_{i}^{2}=\left(\sum_{i} W_{i}\right)^{2}-2 \sum_{i<j} W_{i} W_{j} \\
\sum_{i} W_{i}^{3}=\left(\sum_{i} W_{i}\right)^{3}-3\left(\sum_{i} W_{i}\right)\left(\sum_{j<k} W_{j} W_{k}\right)+3 \sum_{i<j<k} \sum_{i} W_{i} W_{j} W_{k} \\
\sum_{i} W_{i}^{4}=\left(\sum_{i} W_{i}\right)^{4}-4\left(\sum_{i} W_{i}\right)^{2}\left(\sum_{j<k} W_{j} W_{k}\right)+4\left(\sum_{i} W_{i}\right)\left(\sum_{j<k<l} \sum_{j} W_{k} W_{l}\right) \\
+2\left(\sum_{i<j} W_{i} W_{j}\right)^{2}-4 \sum \sum_{i<j<k<l} \sum W_{i} W_{j} W_{k} W_{l} \\
\sum_{i} W_{i}^{5}=\left(\sum_{i} W_{i}\right)^{5}-5\left(\sum_{i} W_{i}\right)^{3}\left(\sum_{j<k} \sum_{j} W_{k}\right)+5\left(\sum_{i} W_{i}\right)^{2}\left(\sum_{j<k<l} \sum W_{j} W_{k} W_{l}\right) \\
+5\left(\sum_{i} W_{i}\right)\left(\sum_{j<k} \sum_{j} W_{k}\right)^{2}-5\left(\sum_{i} W_{i}\right)\left(\sum \sum_{j<k<l<m} \sum_{j} W_{j} W_{k} W_{l} W_{m}\right) \\
-5\left(\sum_{i<j} W_{i} W_{j}\right)\left(\sum_{k<l<m} \sum_{i} W_{k} W_{l} W_{m}\right)+5 \sum \sum_{i<j<k<l<m} \sum W_{i} W_{j} W_{k} W_{l} W_{m}
\end{gathered}
$$




$$
\begin{aligned}
& \sum W_{i}^{6}=\left(\sum_{i} W_{i}\right)^{6}-6\left(\sum_{i} W_{i}\right)^{4}\left(\sum_{j} \sum_{k} W_{j} W_{k}\right)+6\left(\sum_{i} W_{i}\right)^{3}\left(\sum_{j} \sum_{k} \sum_{<l} W_{j} W_{k} W_{l}\right) \\
& +9\left(\sum_{i} W_{i}\right)^{2}\left(\sum_{j} \sum_{k} W_{j} W_{k}\right)^{2}-6\left(\sum_{i} W_{i}\right)^{2}\left(\sum_{j} \sum_{k} \sum_{<l} \sum_{<m} W_{j} W_{k} W_{l} W_{m}\right) \\
& -12\left(\sum_{i} W_{i}\right)\left(\sum_{j} \sum_{k} W_{j} W_{k}\right)\left(\sum_{l<m} \sum_{i} \sum_{n} W_{l} W_{m} W_{n}\right) \\
& +6\left(\sum_{i} W_{i}\right)\left(\sum_{j<} \sum_{k} \sum_{l} \sum_{<} \sum_{<n} W_{j} W_{k} W_{l} W_{m} W_{n}\right) \\
& -2\left(\sum_{i} \sum_{j} W_{i} W_{j}\right)^{3}+6\left(\sum_{i<j} \sum_{j} W_{i} W_{\jmath}\right)\left(\sum_{k} \sum_{l<m} \sum_{i<n} \sum_{k} W_{l} W_{m} W_{n}\right) \\
& \left.+3\left(\sum_{i} \sum_{j} \sum_{k} W_{i} W_{j} W_{k}\right)^{2}-6 \sum_{i<j} \sum_{j<k} \sum_{k} \sum_{l<} \sum_{m} \sum_{<n} W_{i} W_{j} W_{k} W_{l} W_{m} W_{n}\right) .
\end{aligned}
$$

These coefficients for the various terms of the polynomial expansion, $\sum_{i<j} \sum_{i} W_{i} W_{j}$, $\sum_{i} \sum_{<j<k} \sum_{i} W_{i} W_{j} W_{k}$, etc., can be obtained in the case of a specific secular determinant and introduced into eq (2) to (6). If the secular equation

$$
\left|E_{i j}-\delta_{i j} W\right|=0
$$

involves a determinant of the $n$th order with nonzero elements along the principal diagonal and along diagonals immediately adjacent to it, then it is possible to represent the coefficients explicitly and introduce them into eq (2) to (6) so as to represent the quantities $\Sigma W_{i}^{m}$ directly in terms of the elements of the determinant. Thus for the symmetric determinant with $E_{i i}=$ $k_{i}, E_{i, i+1}=E_{i+1, i}=a_{i}, E_{i, i+2}=E_{i+2, i}=b_{i}, E_{i, i+3}=E_{i+3, i}=c_{i}$, and with all other elements equal to zero, one may with considerable algebra transform from the previous equations to the following:

$$
\begin{gathered}
\sum_{i} W_{i}=\sum_{i} k_{i} \\
\sum_{i} W_{i}^{2}=\sum_{i=1}^{n} k_{i}^{2}+2 \sum_{i=1}^{n-1} a_{i}^{2}+2 \sum_{i=1}^{n-2} b_{i}^{2}+2 \sum_{i=1}^{n-3} c_{i}^{2} \\
\sum_{i} W_{i}^{3}=\sum_{i=1}^{n} k_{i}^{3}+3 \sum_{i=1}^{n-1} a_{i}^{2}\left(k_{i}+k_{i+1}\right)+3 \sum_{i=1}^{n-2} b_{i}^{2}\left(k_{i}+k_{i+2}\right)+3 \sum_{i=1}^{n-3} c_{i}^{2}\left(k_{i}+k_{i+3}\right) \\
+6 \sum_{i=1}^{n-2} a_{i} a_{i+1} b_{i}+6 \sum_{i=1}^{n-3} a_{i} b_{i+1} c_{i}+6 \sum_{i=1}^{n-3} a_{i+2} b_{i} c_{i},
\end{gathered}
$$

including terms involving $a$ 's, $b$ 's and $c$ 's. If the $c$ 's are zero, then

$$
\begin{aligned}
\sum_{i} W_{i}^{4}= & \sum_{i=1}^{n} k_{i}^{4}+4 \sum_{i=1}^{n-1} a_{i}^{2}\left(k_{i}^{2}+k_{i} k_{i+1}+k_{i+1}^{2}\right)+4 \sum_{i=1}^{n-2} b_{i}^{2}\left(k_{i}^{2}+k_{i} k_{i+2}+k_{i+2}^{2}\right) \\
& +8 \sum_{i=1}^{n-2} a_{i} a_{i+1} b_{i}\left(k_{i}+k_{i+1}+k_{i+2}\right)+2 \sum_{i=1}^{n-1} a_{i}^{4}+2 \sum_{i=1}^{n-2} b_{1}^{4}+4 \sum_{i=1}^{n-2} a_{i}^{2} a_{i+1}^{2}+4 \sum_{i=1}^{n-3} a_{i}^{2} b_{i+1}^{2} \\
& +4 \sum_{i=1}^{n-2} a_{i}^{2} b_{i}^{2}+4 \sum_{i=1}^{n-2} a_{i+1} b_{i}^{2}+4 \sum_{i=1}^{n-3} a_{i+2}^{2} b_{i}^{2}+8 \sum_{i=1}^{n-3} a_{i} a_{i+2} b_{i} b_{i+1}+4 \sum_{i=1}^{n-4} b_{i}^{2} b_{i+2}^{2},
\end{aligned}
$$

and if the $b$ 's also are zero

$$
\begin{aligned}
\sum_{i} W_{i}^{5}=\sum_{1}^{n} k_{i}^{5}+5 \sum a_{i}^{2}\left(k_{i}^{3}+k_{i}^{2} k_{i+1}+k_{i} k_{i+1}^{2}\right. & \left.+k_{i+1}^{3}\right) \\
& +5 \sum_{1}^{n-1} a_{i}^{4}\left(k_{i}+k_{i+1}\right)+5 \sum_{1}^{n-2} a_{i}^{2} a_{i+1}^{2}\left(k_{i}+2 k_{i+1}+k_{i+2}\right)
\end{aligned}
$$


and

$$
\begin{aligned}
\sum_{i} W_{i}^{6}= & \sum_{1}^{n} k_{i}^{6}+6 \sum_{1}^{n-1} a_{i}^{2}\left(k_{i}^{4}+k_{i}^{3} k_{i+1}+k_{i}^{2} k_{i+1}^{2}+k_{i} k_{i+1}^{3}+k_{i+1}^{4}\right)+3 \sum_{1}^{n-1} a_{i}^{4}\left(3 k_{i}^{2}+4 k_{i} k_{i+1}+3 k_{i+1}^{2}\right) \\
& +6 \sum_{1}^{n-2} a_{i}^{2} a_{i+1}^{2}\left(k_{i}^{2}+2 k_{i} k_{i+1}+k_{i} k_{i+2}+3 k_{i+1}^{2}+2 k_{i+1} k_{i+2}+k_{i+2}^{2}\right) \\
& +2 \sum_{1}^{n-1} a_{i}^{6}+6 \sum_{1}^{n-2} a_{i}^{2} a_{i+1}^{4}+6 \sum_{1}^{n-2} a_{i}^{4} a_{i+1}^{2}+6 \sum_{1}^{n-3} a_{i}^{2} a_{i+1}^{2} a_{i+2}^{2} .
\end{aligned}
$$

Equations (8) to (13) may also be obtained by a matrix method with less effort. This is based on a theorem of Borchardt [1] ${ }^{1}$ and of Sylvester [2] which states that if $A$ is a matrix then the characteristic roots of $A^{n}$ are the $n$th powers of the characteristic roots of $A$. Then if $H$ is the energy matrix, the sum of $n$th powers of roots $W_{i}$ of the secular equation is given by the trace of the $n$th power of the matrix $H$ :

$$
\sum_{i} W_{i}^{n}=\operatorname{trace} H^{n}
$$

so that eq (1) may also be written as

$$
\sum_{i} e^{-W_{i} / k T}=\operatorname{trace} e^{-H / k T}
$$

and, in effect, include all the results of eq (8) to (13).

\section{Application of the Method}

\begin{tabular}{|c|c|c|c|c|c|}
\hline$W_{1}^{0}-W$ & $W_{12}$ & 0 & 0 & . & . \\
\hline$W_{21}$ & $W_{2}^{0}-W$ & $W_{23}$ & 0 & . & . \\
\hline 0 & $W_{32}$ & $W_{3}^{0}-W$ & $W_{34}$ & . & . \\
\hline 0 & 0 & $W_{43}$ & $W_{4}^{0}-W$ & . & . \\
\hline . & $\cdot$ & $\cdot$ & . & · & · \\
\hline . & . & . & . & . & · \\
\hline . & . & . & . & . & . \\
\hline
\end{tabular}

Among cases in which molecular energy is given by a secular equation are resonances of the Darling-Dennison type and the Fermi type. In the case found by Darling and Dennison [3] for the $\mathrm{H}_{2} \mathrm{O}$ molecule, occurring also in some other cases, the energy. $W$ is given by the secular equation

where the energies for the individual interacting levels would have been $W_{i}^{\circ}$ if the perturbation had been negligible. The magnitude of the perturbation is indicated by the off-diagonal terms

$$
W_{i, i+1}=W_{i+1, i}=W_{v_{1}-2, v_{2}, v_{3}+2}^{v_{1}, \ell_{2}, v_{3}}=\frac{1}{2} \gamma \sqrt{v_{1}\left(v_{1}-1\right)\left(v_{3}+1\right)\left(v_{3}+2\right)} .
$$

Any state indicated by $v_{1}, v_{2}, v_{3}$ with $v_{1}>1$ is perturbed appreciably by a state $v_{1}-2, v_{2}, v_{3}+2$, because of the approximate equality of $\nu_{1}$ and $\nu_{3}$. For higher quantum numbers, large groups of levels will be perturbing each other, and the secular equation will involve a secular determinant of correspondingly high order as indicated by eq (16). In the discussion which follows, the number of levels perturbing each other in a single group will be indicated by $n$, which will also be the order of the determinant. The mean value of the energy for the group of levels expressed as wave numbers will be indicated as $G$. The increment in the vibrational partition function due to the presence of the perturbation for a group of interacting levels is given by

$$
\Delta Q=e^{-h c G / k T}\left[-\frac{h c}{k T} \Delta \sum_{i} W_{\imath}+\frac{1}{2}\left(\frac{h c}{k T}\right)^{2} \Delta \sum_{i} W_{i}^{2}-\frac{1}{6}\left(\frac{h c}{k T}\right)^{3} \Delta \sum_{i} W_{i}^{3} \ldots\right] .
$$

${ }^{1}$ Figures in brackets indicate the literature references at the end of this paper. 
According to the formulas derived and the definition of $G$, the various increments in the sums of powers of the energy $W$ measured from the mean value $G$ will be given by

$$
\left.\begin{array}{l}
\Delta \sum_{i} W_{i}=0 \\
\Delta \sum_{i} W_{i}^{2}=2 \sum_{i} W_{i, i+1}^{2} \\
\Delta \sum_{i} W_{i}^{3}=3 \sum_{i} W_{i, i+1}^{2}\left(W_{i}^{\circ}+W_{i+1}^{\circ}\right), \text { etc. }
\end{array}\right\}
$$

In the remainder of the treatment for this case of resonance, only the effect of $\Delta \sum_{i} W_{i}^{\circ}$ on the partition function and derived quantities will be considered. There is a basis for regarding higher order effects, as of $\Delta \sum_{i} W_{i}^{3}$, etc., as negligible. The levels which are involved in a given perturbing set have the same value of $v_{1}+v_{3}$ and are subject to the additional restriction that either $v_{1}$ or $v_{3}$ is only odd or only even within a single group. There are thus four cases to consider:

I. $v_{1}$ ranges from $2 n-2$ to 0 while $v_{3}$ ranges from 0 to $2 n-2$.

II. $v_{1}$ ranges from $2 n-1$ to 1 while $v_{3}$ ranges from 0 to $2 n-2$.

III. $v_{1}$ ranges from $2 n-2$ to 0 while $v_{3}$ ranges from 1 to $2 n-1$.

IV. $v_{1}$ ranges from $2 n-1$ to 1 while $v_{3}$ ranges from 1 to $2 n-1$.

These four cases give, respectively, for $W_{i, i+1}^{2}$

$$
\begin{aligned}
& \text { I. } \quad W_{i, i+1}^{2}=\frac{\gamma^{2}}{4}(2 n-2 i)(2 n-2 i-1)(2 i-1)(2 i) \\
& \text { II. } \quad W_{i, i+1}^{2}=\frac{\gamma^{2}}{4}(2 n+1-2 i)(2 n-2 i)(2 i-1)(2 i) \\
& \text { III. } \quad W_{i, i+1}^{2}=\frac{\gamma^{2}}{4}(2 n-2 i)(2 n-2 i-1)(2 i)(2 i+1) \\
& \text { IV. } \quad W_{1, i+1}^{2}=\frac{\gamma^{2}}{4}(2 n+1-2 i)(2 n-2 i)(2 i)(2 i+1),
\end{aligned}
$$

where in each case $i$ ranges from 1 to $n-1$. The evaluation $\sum_{i=1}^{n-1} W_{i, i+1}^{2}$ is quite straightforward and gives for these cases, respectively,

$$
\begin{aligned}
& \frac{\gamma^{2}}{4} \cdot \frac{2 n(n-1)(n+1)}{15}\left(4 n^{2}-10 n+9\right) \\
& \frac{\gamma^{2}}{4} \cdot \frac{2 n(n-1)(n+1)}{15}(2 n-1)(2 n+1) \\
& \frac{\gamma^{2}}{4} \cdot \frac{2 n(n-1)(n+1)}{15}(2 n-1)(2 n+1)
\end{aligned}
$$

and

$$
\frac{\gamma^{2}}{4} \cdot \frac{2 n(n-1)(n+1)}{15}\left(4 n^{2}+10 n+9\right)
$$

Considering the molecular vibrational energy as if it were due to harmonic oscillators having frequencies $\omega_{1}$ and $\omega_{3}$ wave numbers, for the two vibrations it is found that $G$, the average energy for the group of $n$ levels is given in the various cases by

$$
\begin{aligned}
& \text { I. } G=(n-1)\left(\omega_{1}+\omega_{3}\right) \\
& \text { II. } G=n \omega_{1}+(n-1) \omega_{3}
\end{aligned}
$$




$$
\begin{aligned}
& \text { III. } G=(n-1) \omega_{1}+n \omega_{3} \\
& \text { IV. } G=n\left(\omega_{1}+\omega_{2}\right)
\end{aligned}
$$

The expressions are now at hand to permit the estimate of the relative increase in the partition function due to the perturbation. Continuing to treat the various cases separately, there are obtained for case I,

$$
\begin{aligned}
\left(\frac{\Delta Q}{Q}\right)_{1} & =\frac{\sum_{n=2} e^{-\frac{h c}{k T}\left(\omega_{1}+\omega_{3}\right)(n-1)}\left(\frac{h c}{k T}\right)^{2} \frac{\gamma^{2}}{4} \frac{2 n(n-1)(n+1)}{15}\left(4 n^{2}-10 n+9\right)}{\sum_{v_{3}=0} \sum_{v_{1}=0} e^{-\frac{h c}{k T} \omega_{1} v_{1}} e^{-\frac{h c}{k T} \omega_{3} v_{3}}} \\
& =\gamma^{2}\left(\frac{h c}{k T}\right)^{2} \frac{\left(1-e^{-\frac{h c}{k T} \omega_{1}}\right)\left(1-e^{-\frac{h c}{k T} \omega_{3}}\right)}{\left[1-e^{-\frac{h c}{k T}\left(\omega_{1}+\omega_{3}\right)}\right]^{6}}\left[e^{-\frac{h c}{k T}\left(\omega_{1}+\omega_{3}\right)}+6 e^{-2 \frac{h c}{k T}\left(\omega_{1}+\omega_{3}\right)}+9 e^{-3 \frac{h c}{k T}\left(\omega_{1}+\omega_{3}\right)}\right],
\end{aligned}
$$

for case II,

$$
\begin{aligned}
\left(\frac{\Delta Q}{Q}\right)_{2} & =\frac{e^{\frac{h c}{k T} \omega_{3}} \sum_{n=2} e^{-\frac{h c}{k T}\left(\omega_{1}+\omega_{3}\right) n}\left(\frac{h c}{k T}\right)^{2} \frac{\gamma^{2}}{4} \frac{2 n\left(n^{2}-1\right)\left(4 n^{2}-1\right)}{15}}{\sum_{v_{3}=0} \sum_{v_{1}=0} e^{-\frac{h c}{k T} \omega_{1} v_{1}} e^{-\frac{h c}{k T} \omega_{3} v_{3}}} \\
& =\gamma^{2}\left(\frac{h c}{k T}\right)^{2} \frac{\left(1-e^{-\frac{h c}{k T} \omega_{1}}\right)\left(1-e^{-\frac{h c}{k T} \omega_{3}}\right) e^{\frac{h c}{k T} \omega_{3}}}{\left[1-e^{-\frac{h c}{k T}\left(\omega_{1}+\omega_{3}\right)}\right]^{6}}\left[3 e^{-2 \frac{h c}{k T}\left(\omega_{1}+\omega_{3}\right)}+10 e^{-3 \frac{h c}{k T}\left(\omega_{1}+\omega_{3}\right)}+3 e^{-4 \frac{h c}{k T}\left(\omega_{1}+\omega_{3}\right)}\right],
\end{aligned}
$$

for case III,

$$
\begin{aligned}
\left(\frac{\Delta Q}{Q}\right)_{3} & =\frac{e^{-\frac{h c}{k T} \omega_{1}} \sum_{n=2} e^{-\frac{h c}{k T}\left(\omega_{1}+\omega_{3}\right) n}\left(\frac{h c}{k T}\right)^{2} \frac{\gamma_{2}^{2}}{4} \frac{2 n\left(n^{2}-1\right)\left(4 n^{2}-1\right)}{15}}{\sum_{v_{1}=0} \sum_{v_{3}=0} e^{-\frac{h c}{k T} \omega_{1} v_{1}} e^{-\frac{h c}{k T} \omega_{3} v_{3}}} \\
& =\gamma^{2}\left(\frac{h c}{k T}\right)^{2} \frac{\left(1-e^{-\frac{h c}{k T} \omega_{1}}\right)\left(1-e^{-\frac{h c}{k T} \omega_{3}}\right) e^{-\frac{h c}{k T} \omega_{1}}}{\left[1-e^{-\frac{h c}{k T}\left(\omega_{1}+\omega_{3}\right)}\right]^{6}}\left[3 e^{-2 \frac{h c}{k T}\left(\omega_{1}+\omega_{3}\right)}+10 e^{-3 \frac{h c}{k T}\left(\omega_{1}+\omega_{3}\right)}+3 e^{-4 \frac{h c}{k T}\left(\omega_{1}+\omega_{3}\right)}\right],
\end{aligned}
$$

and for case IV,

$$
\begin{aligned}
\left(\frac{\Delta Q}{Q}\right)_{4} & =\frac{\sum_{n=2} e^{-\frac{h c}{k T}\left(\omega_{1}+\omega_{3}\right) n}\left(\frac{h c}{k T}\right)^{2} \frac{\gamma^{2}}{4} \frac{2 n\left(n^{2}-1\right)}{15}\left(4 n^{2}+10 n+9\right)}{\sum_{v_{1}=0} \sum_{v_{3}=0} e^{-\frac{h c}{k T} \omega_{1} v_{1}} e^{-\frac{h c}{k T} \omega_{3} v_{3}}} \\
& =\gamma^{2}\left(\frac{h c}{k T}\right)^{2} \frac{\left(1-e^{-\frac{h c}{k T} \omega_{1}}\right)\left(1-e^{-\frac{h c}{k T} \omega_{3}}\right)}{\left[1-e^{-\frac{h c}{k T}\left(\omega_{1}+\omega_{3}\right)}\right]^{6}}\left[9 e^{-2 \frac{h c}{k T}\left(\omega_{1}+\omega_{3}\right)}+6 e^{-3 \frac{h c}{k T}\left(\omega_{1}+\omega_{3}\right)}+e^{-4 \frac{h c}{k T}\left(\omega_{1}+\omega_{3}\right)}\right] .
\end{aligned}
$$


Then, in lowest order of approximation, the total effect of the perturbation is indicated by the sum of the separate $(\Delta Q / Q)_{i}$ for the four cases, to give as the approximate value of $\Delta \ln Q$

$$
\begin{aligned}
\Delta \ln Q=\sum_{i}(\Delta Q / Q)_{i}= & \gamma^{2}\left(\frac{h c}{k T}\right)^{2} \frac{\left(1-e^{-\frac{h c}{k T} \omega_{1}}\right)\left(1-e^{-\frac{h c}{k T} \omega_{3}}\right)}{\left[1-e^{-\frac{h c}{k T}\left(\omega_{1}+\omega_{3}\right)}\right]^{6}}\left[e^{-\frac{h c}{k T}\left(\omega_{1}+\omega_{3}\right)}\right. \\
& +15 e^{-2 \frac{h c}{k T}\left(\omega_{1}+\omega_{3}\right)}+15 e^{-3 \frac{h c}{k T}\left(\omega_{1}+\omega_{3}\right)}+e^{-4 \frac{h c}{k T}\left(\omega_{1}+\omega_{3}\right)} \\
+ & \left.\left(e^{-\frac{h c}{k T} \omega_{1}}+e^{-\frac{h c}{k T} \omega_{3}}\right)\left(3 e^{-\frac{h c}{k T}\left(\omega_{1}+\omega_{3}\right)}+10 e^{-2 \frac{h c}{k T}\left(\omega_{1}+\omega_{3}\right)}+3 e^{-3 \frac{h c}{k T}\left(\omega_{1}+\omega_{3}\right)}\right)\right] .
\end{aligned}
$$

If the approximate equality of $\omega_{1}$ and $\omega_{3}$ is now taken into account, then letting $u=h c \omega / k T$ represent either $h c \omega_{1} / k T$ or $h c \omega_{3} / k T$ or their average value, it is found that

$$
\begin{aligned}
\Delta \ln Q & =\frac{\gamma^{2}}{\omega^{2}} \frac{u^{2}\left(1-e^{-u}\right)^{2}}{\left(1-e^{-2 u}\right)^{6}}\left[e^{-2 u}+6 e^{-3 u}+15 e^{-4 u}+20 e^{-5 u}+15 e^{-6 u}+6 e^{-7 u}+e^{-8 u}\right] \\
& =\frac{\gamma^{2}}{\omega^{2}} \frac{u^{2} e^{-2 u}}{\left(1-e^{-u}\right)^{4}} .
\end{aligned}
$$

This is the approximate contribution to the Gibbs free-energy function, $-F^{\circ} / R T$, due to the effect of the perturbation. The corresponding contributions to $H^{\circ} / R T$ and $C_{p}^{\circ} / R$ are $-u \frac{d}{d u} \Delta \ln Q$ and $u^{2} \frac{d^{2}}{d u^{2}} \Delta \ln Q$, which give

and

$$
\Delta\left(H^{\circ} / R T\right)=\frac{\gamma^{2}}{\omega^{2}} u^{2} \frac{e^{-2 u}}{\left(1-e^{-u}\right)^{4}} \cdot 2\left\{u \frac{\left(1+e^{-u}\right)}{\left(1-e^{-u}\right)}-1\right\}
$$

$$
\Delta\left(C_{p}^{\circ} / R\right)=\frac{\gamma^{2}}{\omega^{2}} u^{2} \frac{e^{-2 u}}{\left(1-e^{-u}\right)^{4}} \cdot 2\left\{1-4 u \frac{\left(1+e^{-u}\right)}{\left(1-e^{-u}\right)}+2 u^{2} \frac{\left(1+3 e^{-u}+e^{-2 u}\right)}{\left(1-e^{-u}\right)^{2}}\right\} .
$$

A value of $\gamma$ of $77.52 \mathrm{~cm}^{-1}$ has recently been given by Benedict [4] with higher order constants and has been used in the present calculation. For the present case, $\omega$ may be taken as about $3,650 \mathrm{~cm}^{-1}$. As should be expected, the total effect is found to be small. This is due largely to the fact that the lowest levels showing this type of resonance are at about $7,300 \mathrm{~cm}^{-1} \mathrm{so}$ that a relatively high temperature is required before the levels in question are appreciably excited. The magnitude of the effect is indicated in table 1 for several temperatures.

One may inquire as to the approximate behavior of the effect in the limiting condition of high temperatures. If one neglects higher order effects in the manner of the foregoing deriva-

\begin{tabular}{|c|c|c|c|}
\hline Temperature & $\Delta\left(-F^{\circ} / R T\right)$ & $\Delta\left(H^{\circ} / R T\right)$ & $\Delta C_{p}^{\circ} / R$ \\
\hline $\begin{array}{l}{ }^{\circ} K \\
1,000 \\
2,000 \\
3,000 \\
4,000 \\
5,000\end{array}$ & $\begin{array}{l}2.6 \times 10^{-7} \\
1.88 \times 10^{-5} \\
0.784 \times 10^{-4} \\
1.75 \times 10^{-4} \\
3.03 \times 10^{-4}\end{array}$ & $\begin{array}{l}24 \times 10^{-7} \\
7.81 \times 10^{-5} \\
2.37 \times 10^{-4} \\
4.52 \times 10^{-4} \\
7.23 \times 10^{-4}\end{array}$ & $\begin{array}{l}206 \times 10^{-7} \\
33.1 \times 10^{-5} \\
8.01 \times 10^{-4} \\
14.2 \times 10^{-4} \\
22.1 \times 10^{-4}\end{array}$ \\
\hline
\end{tabular}
tion, which would neglect an appreciable relative effect due to anharmonicity, then when the functions are expanded in increasing powers of $u$, the leading term gives the approximate values

or

$$
-\Delta F^{\circ} / R T \approx \frac{\gamma^{2}}{\omega^{2} u^{2}}, \Delta H^{\circ} / R T \approx 2 \frac{\gamma^{2}}{\omega^{2} u^{2}}, \text { and } \Delta C_{p}^{\circ} / R=6 \frac{\gamma^{2}}{\omega^{2} u^{2}}
$$

TABLE 1. Effect of Darling-Dennison resonance on thermodynamic functions for $\mathrm{H}_{2} \mathrm{O}$

$$
-\Delta F^{\circ} / R T \approx \frac{\gamma^{2}}{\omega^{4}}\left(\frac{k T}{h c}\right)^{2}, \Delta H^{\circ} / R T \approx 2 \frac{\gamma^{2}}{\omega^{4}}\left(\frac{k T}{h c}\right)^{2} \quad \text { and } \quad \Delta C_{p}^{\circ} / R \approx 6 \frac{\gamma^{2}}{\omega^{4}}\left(\frac{k T}{h c}\right)^{2}
$$


The quadratic power of temperature is a result of the fourth power dependence of $\Delta \Sigma W_{i}^{2}$ upon the quantum numbers, which supplies a fourth power of $T$ as a factor in the summation. This is cut down to the second power by the inverse second power in the coefficient of this term.

Another case of resonating levels for which details have been worked out is that of Fermi resonance in the case of $\mathrm{CO}_{2}$. While the effect of the perturbation upon the thermodynamic functions can be expected to be fairly small in this case also, since the perturbation, as in the previous case, involves the displacement of some levels upward and some downward in such a way as to nearly compensate, it is nevertheless a larger effect than in the case of $\mathrm{H}_{2} \mathrm{O}$, mainly because the energy of the lowest resonating levels is near $1330 \mathrm{~cm}^{-1}$ for $\mathrm{CO}_{2}$ as compared with the $7,300 \mathrm{~cm}^{-1}$ for $\mathrm{H}_{2} \mathrm{O}$, so that comparable excitation takes place at a lower temperature.

It was shown by Dennison [5] and Adel and Dennison [6] that a satisfactory representation of the $\mathrm{CO}_{2}$ spectrum could be obtained if account were taken of perturbations within groups of levels with equal values of $l$, the azimuthal quantum number, and with equal values of $2 v_{1}+v_{2}$. Then the effect of the perturbation within a group of $n$ levels of this kind is indicated by the shifts of roots of such a secular equation as (16) with corresponding effects on the thermodynamic functions indicated in terms of the partition function according to eq (18) and (19). In each group of $n$ levels, the value of $v_{1}$ ranges from zero to $n-1$, with $v_{2}$ going from its maximum value to its minimum value which is equal to $|l|$. The number of levels interacting within one group is given by $n=\frac{1}{2}(V-|l|)-1$. The elements of the determinant which are adjacent to the main diagonal were presented by Dennison in a form which can be so converted as to indicate that

$$
W_{i, i+1}^{2}=W_{0}^{2} i(n-1)(V+2-n-i),
$$

where $i$ ranges from 1 to $n-1$. For the evaluation of the principal contribution to the partition function according to eq (18), there is needed a knowledge of the distribution of the unperturbed vibrational levels. It is assumed that these are given in the somewhat unconventional form

$$
G_{v}=\sum \omega_{i} v_{i}+\sum X_{i i} v_{i}\left(v_{i}-1\right)+\sum X_{i j} v_{i} v_{j}
$$

Then the average value of $G$ for a given group of levels is given by

$$
\begin{aligned}
G=\omega_{2} V+\omega_{3} v_{3}+\omega_{n}(n-1)+X_{22}\left(V^{2}-V\right)+ & X_{33}\left(v_{3}^{2}-v_{3}\right)+X_{n n}(n-1)^{2} \\
& +X_{23} V v_{3}+X_{V n} V(n-1)+X_{n 3}(n-1) v_{3},
\end{aligned}
$$

where

$$
\begin{aligned}
\omega_{n} & =\frac{1}{2} \omega_{1}-\omega_{2}-\frac{1}{3} X_{11}+\frac{5}{3} X_{22}-\frac{1}{3} X_{12} \\
X_{n n} & =\frac{1}{3} X_{11}+\frac{4}{3} X_{22}-\frac{2}{3} X_{12} \\
X_{v n} & =\frac{1}{2} X_{12}-2 X_{22} \\
X_{n 3} & =\frac{1}{2} X_{13}-X_{23} .
\end{aligned}
$$

By simple summation,

$$
\sum_{i=1}^{\mathrm{n}-1} W_{i, i+1}^{2}=W_{0}^{2} \frac{n\left(n^{2}-1\right)}{2}\left[\frac{1}{3}(V+2)-\frac{1}{2} n\right]
$$

and for the next term of (18)

$$
\sum_{i=1}^{\mathrm{n}-1} W_{i, i+1}^{2}\left(W_{i}^{0}+W_{i+1}^{0}\right)=W_{0}^{2} \frac{n\left(n^{2}-1\right)\left(4-n^{2}\right)}{30}\left[\omega_{n}-\delta_{v} V-X_{n 3} v_{3}\right],
$$


where $\delta_{v}=\frac{1}{3} X_{11}-\frac{2}{3} X_{22}-\frac{1}{6} X_{12}$. After multiplying by the exponential Boltzmann factor for the average vibrational energy, the summation over $V$ and $n$ follow, but with the requirement that half values of the summand are to be used when $V$ has its lowest value, $2(n-1)$, in order to take account of the absence of $l$ degeneracy for $l=0$. The choice of doing this rather than multiplying by 2 for $l \neq 0$ comes about as the result of the fact that half of the rotational levels are absent due to the symmetry of the molecule and the zero nuclear spin for oxygen. Then this is compared with a total approximate $Q$ similarly omitting any factor for $l$ doubling. If the effects for anharmonicity are disregarded, the leading term is obtained as

$$
\frac{\Delta Q}{Q} \approx \frac{1}{2}\left(\frac{h c}{k T}\right)^{2} \frac{W_{0}^{2} e^{-\frac{2 h c \omega_{2}}{k T}}\left(1-e^{-\frac{h c \omega_{1}}{k T}}\right)}{\left(1-e^{-\frac{2 h c \omega_{2}}{k T}}\right)^{2}\left(1-e^{-\frac{h c \omega_{2}}{k T}}\right)^{2}} .
$$

The effect of Fermi resonance, using $u=h c \omega_{2} / k T$ and disregarding the distinction between $\omega_{1}$ and $2 \omega_{2}$, is thus given approximately [7] by

$$
\begin{gathered}
\Delta\left(-F^{\circ} / R T\right)=\frac{1}{2} \frac{W_{0}^{2}}{\omega_{2}^{2}} \frac{u^{2} e^{-2 u}}{\left(1-e^{-2 u}\right)\left(1-e^{-u}\right)^{2}}, \\
\Delta H^{\circ} / R T=\Delta\left(-F^{\circ} / R T\right) \cdot 2\left[u\left(1+\frac{e^{-u}}{1-e^{-u}}+\frac{e^{-2 u}}{1-e^{-2 u}}\right)-1\right],
\end{gathered}
$$

and

$$
C_{p}^{0} / R=\Delta\left(-F^{\circ} / R T\right) \cdot 2\left\{2\left[u\left(1+\frac{e^{-u}}{1-e^{-u}}+\frac{e^{-2 u}}{1-e^{-2 u}}\right)-1\right]^{2}-u^{2}\left[\frac{e^{-u}}{\left(1-e^{-u}\right)^{2}}+\frac{2 e^{-2 u}}{\left(1-e^{-2 u}\right)^{2}}\right]-1\right\} .
$$

The effect can be obtained in more detail by including an additional contribution from eq (46). Using $u_{i}=h c \omega_{i} / k T$ and using $\omega_{1}=2 \omega_{2}$ after the first term, eq (48) is replaced by

$\Delta \ln Q=\frac{1}{2}\left(\frac{h c}{k T}\right)^{2} \frac{W_{0}^{2} e^{-2 u_{2}}\left(1-e^{-u_{1}}\right)}{\left(1-e^{-2 u_{2}}\right)^{2}\left(1-e^{-u_{2}}\right)^{2}}$

$$
\begin{aligned}
& -\left(\frac{h c}{k T}\right)^{3} W_{0}^{2}\left\{\frac{\omega_{n}}{2\left(1-e^{-2 u_{2}}\right)^{4}}\left[e^{-2 u_{2}}+2 e^{-3 u_{2}}+6 e^{-4 u_{2}}+6 e^{-5 u_{2}}+3 e^{-6 u_{2}}\right]\right. \\
& +\frac{X_{n n}}{2\left(1-e^{-2 u_{2}}\right)^{5}}\left[e^{-2 u_{2}}+2 e^{-3 u_{2}}+17 e^{-4 u_{2}}+20 e^{-5 u_{2}}+33 e^{-6 u_{2}}+18 e^{-7 u_{2}}+9 e^{-8 u_{2}}\right] \\
& +\frac{X_{V n}}{\left(1-e^{\left.-2 u_{2}\right)^{5}}\right.}\left[e^{-2 u_{2}}+4 e^{-3 u_{2}}+19 e^{-4 u_{2}}+30 e^{-5 u_{2}}+39 e^{-6 u_{2}}+24 e^{-7 u_{2}}+9 e^{-8 u_{2}}\right]
\end{aligned}
$$

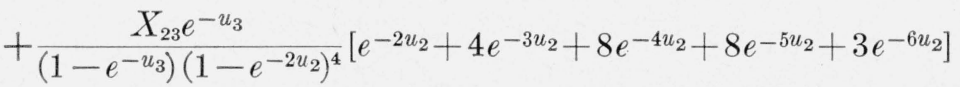

$$
\begin{aligned}
& +\frac{X_{n 3} e^{-u_{3}}}{2\left(1-e^{-u_{3}}\right)\left(1-e^{-2 u_{2}}\right)^{4}}\left[e^{-2 u_{2}}+2 e^{-3 u_{2}}+6 e^{-4 u_{2}}+6 e^{-5 u_{2}}+3 e^{-6 u_{2}}\right]
\end{aligned}
$$

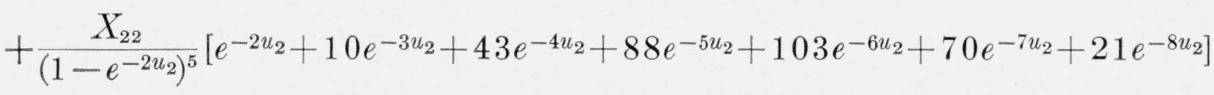

$$
\begin{aligned}
& -\frac{B+g_{22}}{6\left(1-e^{-2 u_{2}}\right)^{5}}\left[e^{-3 u_{2}}+8 e^{-4 u_{2}}+41 e^{-5 u_{2}}+104 e^{-6 u_{2}}+119 e^{-7 u_{2}}+92 e^{-8 u_{2}}+19 e^{-9 u_{2}}\right] \\
& -\frac{X_{11} e^{-6 u_{2}}}{\left(1-e^{-2 u_{2}}\right)^{3}\left(1-e^{\left.-u_{2}\right)^{2}}\right.}-\frac{3 X_{22} e^{-4 u_{2}}}{\left(1-e^{-2 u_{2}}\right)\left(1-e^{-u_{2}}\right)^{4}}-\frac{X_{12} e^{-5 u_{2}}}{\left(1-e^{-2 u_{2}}\right)^{2}\left(1-e^{-u_{2}}\right)^{3}} \\
& \left.-\frac{X_{13} e^{-u_{3}} e^{-4 u_{2}}}{2\left(1-e^{-u_{3}}\right)\left(1-e^{-2 u_{2}}\right)^{2}\left(1-e^{\left.-u_{2}\right)^{2}}\right.}-\frac{X_{23} e^{-u_{3}} e^{-3 u_{2}}}{\left(1-e^{-u_{3}}\right)\left(1-e^{-2 u_{2}}\right)\left(1-e^{-u_{2}}\right)^{3}} \cdot\right\}
\end{aligned}
$$


A calculation was made for $\mathrm{CO}_{2}$ using as values for the constants (in $\mathrm{cm}^{-1}$ )

$$
\begin{aligned}
& \omega_{1}=1342.86 \quad \omega_{2}=666.27 \quad \omega_{3}=2349.15 \\
& X_{11}=-2.20 \quad X_{22}=-0.75 \quad X_{33}=-12.46 \\
& X_{12}=3.76 \quad X_{13}=-18.54 \quad X_{23}=-12.48
\end{aligned}
$$

with $B+g_{22}=1.42$ and $W_{0}=51.01 \mathrm{~cm}^{-1}$, giving $\omega_{n}=3.39, X_{n n}=-4.24, X_{V n}=3.38$, and $X_{n 3}=3.21$. With these constants, the magnitude of the effect as given by eq (48) is indicated in table 2 as the first column under each heading while the combined effect consistent with eq (52) is shown as the second column under each of the headings, $\Delta\left(-F^{\circ} / R T\right), \Delta\left(H^{\circ} / R T\right)$ and $\Delta C_{p}^{\circ} / R$. The entire effect for $\mathrm{CO}_{2}$ is rather small and in many computations could be neglected. For elevated temperature, the leading term in this case is roughly linear in the absolute temperature rather than quadratic as was found to be the case for $\mathrm{H}_{2} \mathrm{O}$. This is due to the essentially cubic dependence of $W_{i, i+1}^{2}$ upon quantum numbers in the present case, as compared with the quartic dependence in the case of $\mathrm{H}_{2} \mathrm{O}$. It is also seen that this simple dependence upon temperature is modified greatly by the effect of anharmonicity. The large effect of anharmonicity at high temperature can be regarded as due to its lowering of entire groups of resonating levels with a resultant increased population in such groups, which provides an increase in thermody. namic effect.

This treatment does not make any adjustment for the effect of resonance on the compositions of states, since the rotational perturbation is quite small, and, in addition, the resonance shifts have compensating effects in first approximation as has been noted in deriving the larger contributions due to perturbation which have been taken into account. They would thus be

\begin{tabular}{|c|c|c|c|c|c|c|}
\hline Temperature & \multicolumn{2}{|c|}{$\Delta\left(-F^{\circ} / R T\right)$} & \multicolumn{2}{|c|}{$\Delta\left(H^{\circ} / R T\right)$} & \multicolumn{2}{|c|}{$\Delta\left(C_{p} / R\right)$} \\
\hline $\begin{array}{l}{ }^{\circ} K \\
200 \\
250 \\
300 \\
400 \\
500\end{array}$ & \multicolumn{2}{|c|}{$\begin{array}{l}5 \times 10^{-6} \\
22 \times 10^{-6} \\
5 \times 10^{-5} \\
18 \times 10^{-5} \\
33 \times 10^{-5}\end{array}$} & \multicolumn{2}{|c|}{$\begin{array}{l}36 \times 10^{-6} \\
12 \times 10^{-5} \\
26 \times 10^{-5} \\
56 \times 10^{-5} \\
85 \times 10^{-5}\end{array}$} & \multicolumn{2}{|c|}{$\begin{array}{l}27 \times 10^{-5} \\
70 \times 10^{-5} \\
11 \times 10^{-4} \\
17 \times 10^{-4} \\
22 \times 10^{-4}\end{array}$} \\
\hline & eq $(49)$ & $\mathrm{eq}(52)$ & $\mathrm{eq}(50)$ & $\mathrm{eq}(52)$ & $\mathrm{eq}(51)$ & $\mathrm{eq}(52)$ \\
\hline $\begin{array}{l}1,000 \\
2,000 \\
4,000 \\
5,000\end{array}$ & $\begin{array}{r}0.0012 \\
.0029 \\
.0045 \\
.0061 \\
.0077\end{array}$ & $\begin{array}{l}0.0012 \\
.0030 \\
.0048 \\
.0067 \\
.0087\end{array}$ & $\begin{array}{l}0.0018 \\
.0033 \\
.0048 \\
.0063 \\
.0078\end{array}$ & $\begin{array}{r}0.0018 \\
.0036 \\
.0056 \\
.0078 \\
.0103\end{array}$ & $\begin{array}{r}0.0033 \\
.0062 \\
.0093 \\
.0124 \\
.0155\end{array}$ & $\begin{array}{r}0.0035 \\
.0073 \\
.0119 \\
.0173 \\
.0233\end{array}$ \\
\hline
\end{tabular}
quite negligible.

TABLE 2. Effect of Fermi resonance on thermodynamic functions for $\mathrm{CO}_{2}$

Equations (49), (50), and (51) also represent approximately the effect of Fermi resonance in $\mathrm{H}_{2} \mathrm{O}$ involving levels for which $2 v_{1}+v_{2}$ is a constant, even though $\nu_{2}$ is not degenerate as for $\mathrm{CO}_{2}$. Benedict [4] gives the matrix element $\left(v_{1}, v_{2}+2 \mid v_{1}+1, v_{2}\right)=\frac{1}{2}\left\{200\left(v_{1}+1\right)\left(v_{2}+2\right)\left(v_{2}+1\right)\right\}^{\frac{1}{2}}$, indicating that $W_{0}^{2}=200 \mathrm{~cm}^{-2}$. A few values for the Fermi resonance effect for $\mathrm{H}_{2} \mathrm{O}$ are given

\begin{tabular}{|c|c|c|c|}
\hline Temperature & $\Delta\left(-F^{\circ} / R T\right)$ & $\Delta\left(H^{\circ} / R T\right)$ & $\Delta C_{p}^{\circ} / R$ \\
\hline $\begin{array}{l}{ }^{\circ} K \\
500 \ldots 00 \\
1,000 \\
3,000000 \\
4,000 \\
5,000\end{array}$ & $\begin{array}{l}8 \times 10^{-8} \\
3 \times 10^{-6} \\
10^{-5} \\
2 \times 10^{-5} \\
3 \times 10^{-5} \\
4 \times 10^{-5}\end{array}$ & $\begin{array}{l}10^{-6} \\
8 \times 10^{-6} \\
2 \times 10^{-5} \\
3 \times 10^{-5} \\
4 \times 10^{-5} \\
4 \times 10^{-5}\end{array}$ & $\begin{array}{l}4 \times 10^{-6} \\
2 \times 10^{-5} \\
4 \times 10^{-5} \\
5 \times 10^{-5} \\
7 \times 10^{-5} \\
9 \times 10^{-5}\end{array}$ \\
\hline
\end{tabular}
in table 3. The effect is much smaller than the Darling-Dennison resonance effect at the higher

TABLE 3. Effect of Fermi resonance on thermodynamic functions for $\mathrm{H}_{2} \mathrm{O}$ 
temperatures although it is the larger at $1,000^{\circ} \mathrm{K}$ and below. Its earlier rise is partly due to the resonance occurring first near $3,400 \mathrm{~cm}^{-1}$ as compared with $7,300 \mathrm{~cm}^{-1}$ for the DarlingDennison resonance. The magnitude of the resonance effects for $\mathrm{H}_{2} \mathrm{O}$ and $\mathrm{CO}_{2}$ are shown in figures 1 and 2, respectively.

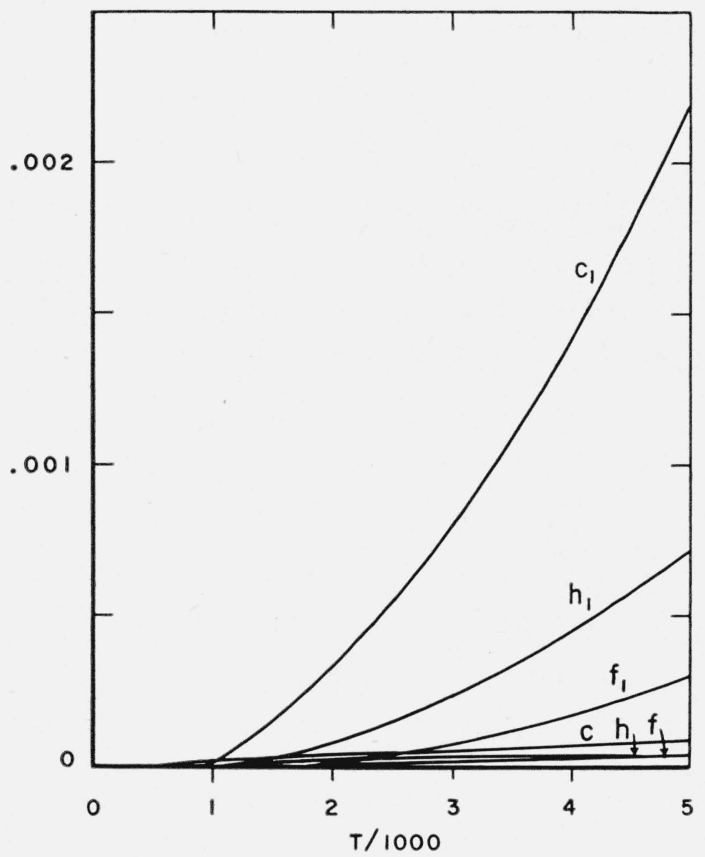

FIGURE 1. Effect of resonance on thermodynamic functions for $\mathrm{H}_{2} \mathrm{O}$.

$\mathrm{c}_{1}, \mathrm{~h}_{1}, \mathrm{f}_{1}$ : Contribution of Darling-Dennison resonance to $C_{F} / R$, Fermi resonance to $C_{p}^{\circ} / R,\left(H^{\circ}-E\right) / R T$, and $-\left(F^{\circ}-E_{\circ}^{\circ}\right) / R T$

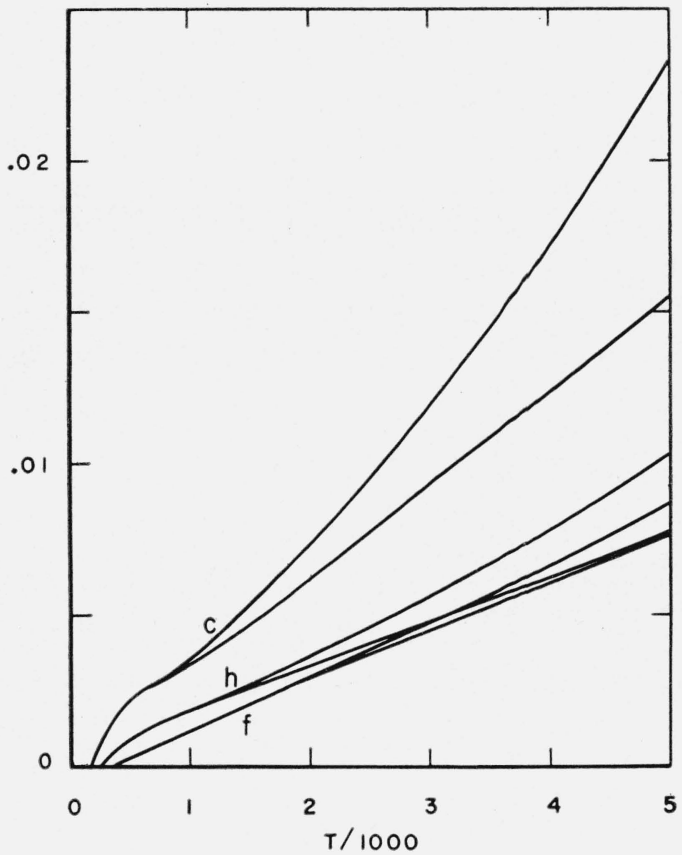

FiguRE 2. Effect of Fermi resonance on thermodynamic functions for $\mathrm{CO}_{2}$.

c, h, f: Contributions to $C \cdot / R,\left(H^{\circ}-E_{0}^{\circ}\right) / R T$, and $-\left(F^{\circ}-E_{0}^{\circ}\right) / R T$. Upper branches with anharmonicity, lower branches neglecting anharmonicity.

\section{Conclusion}

A useful method of calculation of thermodynamic effects for levels defined by a secular determinant equation has been demonstrated.

Many other instances with similar perturbations are known and their effects on thermodynamic functions should be calculable by similar methods if the theoretical description of the perturbation has been worked out and the spectra fitted in the detail that has been achieved for $\mathrm{H}_{2} \mathrm{O}$ and $\mathrm{CO}_{2}$. In particular, $\mathrm{C}_{2} \mathrm{H}_{2}$ gives an example of Darling-Dennison resonance for which eq (37), (38), and (39) would apply. Other molecules with Fermi resonance similar to that for $\mathrm{CO}_{2}$ to which eq (49), (50) and (51) apply include $\mathrm{CS}_{2}, \mathrm{C}_{2} \mathrm{H}_{6}, \mathrm{C}_{2} \mathrm{D}_{6}$ and $\mathrm{N}_{2} \mathrm{O}$.

\section{References}

(1) C. W. Borchardt, J. Reine Angew. Math. 30, 38 (1846) ; J. Math. Pures Appl. [I] 12, 50 (1847).

(2) J. J. Sylvester, Nouv. Ann. math. 11, 439 (1852).

(3) B. T. Darling and D. M. Dennison, Phys. Rev. 57, 128 (1940).

(4) W. S. Benedict, unpublished data.

(5) D. M. Dennison, Phys. Rev. 41, 304 (1932).

(6) A. Adel and D. M. Dennison, Phys. Rev. 43, 716 (1933).

(7) Harold W. Woolley, The effect of Fermi resonance on the thermodynamic functions of gaseous $\mathrm{CO}_{2}, \mathrm{Phys}$ Rev. 72, 183 (1947).

Washin GTON, February 11, 1954. 